\title{
InterferênCIA dA PALha de CANA-De-AÇÚCAR (Saccharum spp.) NA EMERGÊnCIA de EspécIes de Plantas Daninhas da Família CONVOLVULACEAE ${ }^{1}$
}

\author{
Sugar Cane (Saccharum spp.) Straw Interference in Emergence of Weed Species of the \\ Convolvulaceae Family
}

AZANIA, A.A.P.M. ${ }^{2}$, AZANIA, C.A.M. ${ }^{2}$, GRAVENA, R. ${ }^{2}$, PAVANI, M.C.M.D. ${ }^{3}$ e PITELLI, R.A. ${ }^{3}$

\begin{abstract}
RESUMO - Este trabalho foi conduzido na Faculdade de Ciências Agrárias e Veterinárias de Jaboticabal-UNESP, com o objetivo de estudar o efeito de diferentes quantidades de palha de cana-de-açúcar deixadas na superfície do solo sobre a emergência de algumas espécies de plantas daninhas pertencentes à família Convolvulaceae. Os tratamentos foram distribuídos no esquema de parcelas subsubdivididas, com a quantidade de palha nas parcelas de 0,5 , 10, 15 e $20 \mathrm{t} \mathrm{ha}^{-1}$, as variedades SP 792233 e RB 835486 nas subparcelas e as espécies de plantas daninhas nas subsubparcelas. Aos 45 dias após semeadura (DAS), a presença de $15 \mathrm{t} \mathrm{ha}^{-1}$ de palha reduziu em 46 e $62 \%$ o número de plantas de $I$. quamoclit e $M$. cissoides, respectivamente. Entretanto, a presença de $20 \mathrm{t} \mathrm{ha}^{-1}$ reduziu em 82, 65, 62, 70, 60 e 88\% o número de plantas de I. quamoclit, I. purpurea, I. grandifolia, I. hederifolia, I. nil e M. cissoides, respectivamente, quando comparadas à ausência de palha.
\end{abstract}

Palavras-chave: cobertura morta, Ipomoea spp., Merremia cissoides.

ABSTRACT - This research was conducted at FCAV of Jaboticabal-UNESP-Brazil, aiming to study the effect of different amounts of sugarcane straw placed on the soil surface on emergence of some weed species of the Convolvulaceae family. The experimental design was a split-split-plot with the straw amounts in the plots $\left(0,5,10,15\right.$ and $\left.20 \mathrm{tha}^{-1}\right)$, sugarcane cultivars (SP 792233 e RB 83 5486) in the split-plots and weeds in the split-split-plots. The presence of $15 t^{h^{-1}}$ of straw reduced in 46 and $62 \%$ the number of I. quamoclit and $\boldsymbol{M}$. cissoides plants, 45 days

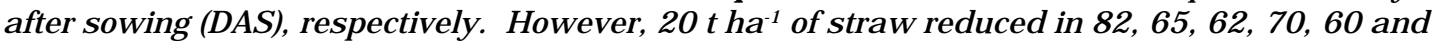
$88 \%$ the number of I. quamoclit, I. purpurea, I. grandifolia, I. hederifolia, I. nil, M. cissoides, respectively, when compared to the absence of straw.

Key words: mulching, Ipomoea spp., Merremia cissoides.

\section{INTRODUÇÃO}

A colheita de cana-crua foi implantada no Brasil há menos de duas décadas, para minimizar os problemas que a colheita tradicional com queima causa ao homem e ao meio ambiente. Por se tratar de uma técnica relativamente nova, os produtores ainda não estão totalmente adaptados à nova realidade. Diante dos diversos problemas enfrentados nas áreas colhidas com cana-crua, foram destacados aqueles relacionados às plantas daninhas, que, em algumas áreas, apresentaram mudanças na composição das espécies infestantes, devido à presença da palha da cana-crua.

A composição da flora infestante e a eficiência do controle de plantas daninhas pela cobertura morta devem ser determinadas pela quantidade, composição, periodicidade da produção e tempo de permanência da cobertura morta na área, que são características que dependem do cultivar, clima e manejo da área (Almeida \& Rodrigues, 1985).

Recebido para publicação em 5/3/2002 e na forma revisada em 7/8/2002.

2 Pós-graduandos em Produção Vegetal, FCAV-UNESP, Via de Acesso Paulo D. Castelane, s/n, 14884-900 Jaboticabal-SP. ${ }^{3}$ Profs. Drs. do Dep. de Biologia Aplicada à Agropecuária da FCAV-UNESP. 
Teasdale et al. (1991) observaram redução de $78 \%$ de algumas espécies de plantas daninhas quando a densidade da cobertura morta foi superior a $300 \mathrm{~g} \mathrm{~m}^{-2}$ e a porcentagem de recobrimento do solo foi superior a $90 \%$. Os autores afirmaram que a cobertura morta pode ter interferido na qualidade da luz e reduzido a germinação das sementes, além de ter impedido fisicamente a emergência das plântulas.

De acordo com Taylorson \& Borthwick (1969) e Fener (1980), a cobertura morta afetou a comunidade infestante não só alterando a quantidade de radiação solar incidente, mas também a qualidade do comprimento das ondas luminosas e a manutenção da temperatura com menores oscilações.

Egley \& Duke (1985) mencionaram que a redução da amplitude térmica da superfície do solo pode interferir de modo decisivo na germinação de muitas espécies. A exigência de maior ou menor amplitude térmica do solo constituiuse no modo mais eficiente de controlar a germinação no solo.

Segundo Pitelli (1995), o efeito físico da cobertura morta também pode reduzir as chances de sobrevivência das plantas daninhas com pouca quantidade de reserva nas sementes, as quais podem não ser suficientes para garantir a sobrevivência da planta no espaço percorrido dentro da cobertura morta, até que tenha acesso à luz e inicie o processo de fotossíntese.

Medina Melendez (1990) observou que a palha de cana-de-açúcar inibiu parcialmente a germinação de sementes de Amaranthus viridis, Galinsoga parviflora, Portulaca oleracea e Lepidium virginicum e totalmente a germinação de sementes de Brachiaria decumbens, Cenchrus echinatus, Ipomoea spp. e Bidens pilosa.

Martins et al. (1999) estudaram os efeitos da cobertura do solo, com quantidades crescentes de palha de cana-de-açúcar até $15 \mathrm{t} \mathrm{ha}^{-1}$, sobre a germinação de algumas das principais plantas daninhas da cultura da cana-de-açúcar no Brasil. Dentre as espécies estudadas, constataram que Ipomoea grandifolia somente sofreu redução na germinação na presença de $15 \mathrm{t} \mathrm{ha}^{-1}$ de palha, havendo estímulos à germinação nas quantidades de 2 a $10 \mathrm{t} \mathrm{ha}^{-1}$ de palha.
As convolvuláceas, principalmente as pertencentes aos gêneros Ipomoea e Merremia, destacam-se dentre as plantas daninhas que podem causar sérios danos à cultura da canade-açúcar, especialmente em áreas de canacrua. Além de competirem com a planta cultivada, podem interferir nas práticas culturais, especialmente na colheita mecanizada, reduzindo sua eficiência. Dessa forma, devido ao crescente aumento das áreas destinadas à colheita mecanizada da cana-crua, foi conduzido o presente trabalho com o objetivo de estudar o efeito de quantidades crescentes de palha de cana-de-açúcar sobre a emergência de Ipomoea spp. e Merremia cissoides.

\section{MATERIAL E MÉTODOS}

O experimento foi conduzido na Faculdade de Ciências Agrárias e Veterinárias de Jaboticabal-UNESP, em área experimental pertencente ao Departamento de Biologia Aplicada à Agropecuária, em molduras de alvenaria construídas sobre o solo, com área de $1,44 \mathrm{~m}^{2} /$ parcela. O substrato utilizado no preenchimento das molduras foi preparado na proporção $2: 1$, sendo terra de textura argilosa e torta de filtro, respectivamente.

A partir dos dados obtidos nos testes de germinação (Tabela 1), semeou-se quantidade de sementes necessária para obter no mínimo 25 plantas de Merremia cissoides, Ipomoea quamoclit, Ipomoea purpurea, Ipomoea grandifolia, Ipomoea hederifolia e Ipomoea nil, aproximadamente a $3 \mathrm{~cm}$ de profundidade e cobertas com palha dos cultivares SP 792233 e RB 835486, nas densidades de 0, 5, 10, 15 e $20 \mathrm{t} \mathrm{ha}^{-1}$. A partir do lote de sementes de cada espécie, foi conduzido teste de germinação, e as sementes não-germinadas foram cortadas ao meio e submetidas, durante 24 horas, ao teste topográfico de tetrazólio a $0,1 \%$, para determinação do percentual de viabilidade do lote.

A temperatura do solo e a quantidade de água foram monitoradas do início ao final da instalação do experimento, sendo o monitoramento da temperatura em 5 e $10 \mathrm{~cm}$ de profundidade e a quantidade de água, através do controle de chuvas e irrigação.

O delineamento experimental utilizado foi em blocos, com cinco tratamentos casualizados 
e cinco repetições, sendo os tratamentos distribuídos no esquema de parcelas subsubdivididas, com as densidades de palha nas parcelas, a palha dos cultivares nas subparcelas e as espécies de plantas daninhas nas subsubparcelas. As parcelas foram constituídas por $1,44 \mathrm{~m}^{2}$, as subparcelas por $0,72 \mathrm{~m}^{2}$ e as subsubparcelas por $0,12 \mathrm{~m}^{2}$. A análise de variância pelo teste $\mathrm{F}$ foi utilizada para avaliar o efeito dos tratamentos sobre as variáveis analisadas; posteriormente, para comparação das médias dos tratamentos, utilizou-se o teste de Tukey a $5 \%$ de probabilidade.

Avaliaram-se a emergência das plantas de cada espécie, aos 15,30 e 45 dias após semeadura (DAS), e a massa seca das plantas, coletadas aos 45 DAS.

\section{RESULTADOS E DISCUSSÃO}

Em laboratório, no teste de germinação realizado nos lotes de sementes, constatou-se que as Ipomoea spp. e Merremia cissoides estavam dormentes. Ocorreu que nenhuma espécie apresentou germinação maior que $30 \%$ e que a viabilidade das sementes não-germinadas foi de $93 \%$ para I. nil, 97\% para I. quamoclit e I. purpurea, $98 \%$ para M. cissoides e 99\% para I. grandifolia e I. hederifolia.

No campo, conforme se pode observar pela Tabela 2, os cultivares RB 835486 e SP 792233 não diferiram estatisticamente; portanto, as diferenças estatísticas observadas para as variáveis avaliadas devem-se principalmente ao aumento das densidades de palha ou a fatores da própria espécie daninha.

Considerando apenas o fator densidade de palha, observa-se que a média do número de plantas daninhas de todas as espécies, para as três épocas avaliadas, diminuiu à medida que se aumentou a densidade de palha. Entretanto, as densidades de 15 e $20 \mathrm{t} \mathrm{ha}^{-1}$ de palha proporcionaram as maiores reduções no número médio de plantas daninhas de todas as espécies, especialmente a densidade de $20 \mathrm{t} \mathrm{ha}^{-1}$ de palha, que, unicamente, diferiu estatisticamente de todas as outras densidades quanto ao número médio de plantas daninhas nas três avaliações, e à massa seca aos 45 DAS (Tabela 2).

Portanto, de acordo com a Tabela 2, quando se considera o número médio de plantas daninhas para cada espécie, independentemente da densidade, observa-se que I. hederifolia foi a espécie que mais se desenvolveu, por apresentar o maior número de indivíduos e acúmulo de massa seca, enquanto $M$. cissoides foi a que menos se desenvolveu, devido ao menor número de indivíduos e acúmulo de massa seca. As plantas daninhas que mais emergiram a camada de palha foram I. hederifolia, I. grandifolia, I. quamoclit, I. nil, M. cissoides e I. purpurea, das quais I. quamoclit, I. nil, M. cissoides e I. purpurea não apresentaram diferenças estatísticas.

As espécies de Ipomoea spp. e M. cissoides nas subsubparcelas diferiram estatisticamente em razão das densidades de palha nas parcelas e não dos diferentes cultivares de cana-de-açúcar utilizados nas subparcelas.

Tabela 1 - Resultados do teste de germinação realizado para as espécies Ipomoea quamoclit, Ipomoea purpurea, Ipomoea grandifolia, Ipomoea hederifolia, Ipomoea nil e Merremia cissoides. Engenheiro Coelho, 1999

\begin{tabular}{|l|c|l|c|c|}
\hline \multicolumn{1}{|c|}{ Nome científico } & Código** & Nome comum** & $\begin{array}{c}\text { Dias para início da } \\
\text { germinação* }\end{array}$ & $\begin{array}{c}\text { Gramas de sementes necessários } \\
\text { para nascer 25 plantas* }\end{array}$ \\
\hline Ipomoea quamoclit & IPOQU & Flor-de-cardeal & 8 & 1,06 \\
Ipomoea purpurea & PHBPU & Corda-de-viola & 8 & 3,57 \\
Ipomoea grandifolia & IAOGR & Corda-de-viola & 8 & 2,03 \\
Ipomoea hederifolia & IPOHF & Corda-de-viola & 7 & 6,25 \\
Ipomoea nil & IPONI & Campainha & 8 & 2,42 \\
Merremia cissoides & MRRCI & Campainha & 8 & 5,00 \\
\hline
\end{tabular}

* Teste de germinação realizado pelo fornecedor das sementes.

** Fonte: Lorenzi (2000). 
Tabela 2 - Resultados da análise de variância para o efeito dos fatores principais sobre o número de plantas e a massa seca das espécies de IPOQU, PHBPU, IAOGR, IPOHF, IPONI e MRRCI em três épocas de avaliação. Jaboticabal, 2000

\begin{tabular}{|c|c|c|c|c|}
\hline \multirow{2}{*}{ Variável } & \multicolumn{3}{|c|}{ Número de plantas - DAS* } & \multirow{2}{*}{$\begin{array}{c}\text { Massa seca } \\
\text { das plântulas aos } 45 \text { DAS } \\
(\mathrm{g})^{*}\end{array}$} \\
\hline & 15 & 30 & 45 & \\
\hline \multicolumn{5}{|l|}{ Densidade (A) - $\left(\mathrm{t} \mathrm{ha}^{-1}\right)$} \\
\hline 0 & $6,06 \mathrm{a}$ & $6,23 \mathrm{a}$ & $6,35 \mathrm{a}$ & $4,51 \mathrm{a}$ \\
\hline 5 & $5,81 \mathrm{ab}$ & $5,90 \mathrm{a}$ & $5,97 \mathrm{a}$ & $4,53 \mathrm{a}$ \\
\hline 10 & $5,12 \mathrm{bc}$ & $5,43 a b$ & $5,61 \mathrm{ab}$ & $4,19 \mathrm{a}$ \\
\hline 15 & $4,68 \mathrm{c}$ & $5,03 \mathrm{~b}$ & $5,14 \mathrm{~b}$ & $4,09 \mathrm{a}$ \\
\hline 20 & $2,73 \mathrm{~d}$ & $3,21 \mathrm{c}$ & $3,33 \mathrm{c}$ & $2,97 \mathrm{~b}$ \\
\hline \multicolumn{5}{|l|}{ Cultivares (B) } \\
\hline $\mathrm{RB}$ & $4,92 \mathrm{a}$ & $5,19 \mathrm{a}$ & $5,32 \mathrm{a}$ & $4,08 \mathrm{a}$ \\
\hline SP & $4,84 \mathrm{a}$ & $5,13 \mathrm{a}$ & $5,23 \mathrm{a}$ & $4,04 \mathrm{a}$ \\
\hline \multicolumn{5}{|l|}{ Espécies (C) } \\
\hline IPOQU & $4,71 \mathrm{c}$ & $5,10 \mathrm{c}$ & $5,26 \mathrm{c}$ & $3,09 \mathrm{c}$ \\
\hline PHBPU & $3,65 \mathrm{~d}$ & $3,79 \mathrm{~d}$ & $3,86 \mathrm{~d}$ & $4,53 a b$ \\
\hline IAOGR & $5,49 \mathrm{~b}$ & $5,78 \mathrm{~b}$ & $5,90 \mathrm{~b}$ & $4,16 \mathrm{~b}$ \\
\hline IPOHF & $6,79 \mathrm{a}$ & $7,28 \mathrm{a}$ & $7,51 \mathrm{a}$ & 5,07 a \\
\hline IPONI & $4,76 \mathrm{c}$ & $4,91 \mathrm{c}$ & $4,98 \mathrm{c}$ & $4,66 a b$ \\
\hline \multirow[t]{2}{*}{ MRRCI } & $3,86 \mathrm{~d}$ & $4,10 \mathrm{~d}$ & $4,16 \mathrm{~d}$ & $2,84 \mathrm{c}$ \\
\hline & \multicolumn{4}{|c|}{$\mathbf{F}$} \\
\hline Blocos & $2,78 \mathrm{NS}$ & $3,11 *$ & $2,00 \mathrm{NS}$ & $2,60 \mathrm{NS}$ \\
\hline Densidade (A) & $60,10 * *$ & $40,10 * *$ & $39,28 * *$ & $18,34 * *$ \\
\hline Cultivares (B) & $0,14 \mathrm{NS}$ & $0,10 \mathrm{NS}$ & $0,17 \mathrm{NS}$ & $0,24 \mathrm{NS}$ \\
\hline Espécies (C) & $83,83 * *$ & $85,37 * *$ & $85,04 * *$ & $36,42 * *$ \\
\hline$A \times B$ & $0,45 \mathrm{NS}$ & $0,75 \mathrm{NS}$ & $0,73 \mathrm{NS}$ & $0,91 \mathrm{NS}$ \\
\hline $\mathrm{A} \times \mathrm{C}$ & $2,34 * *$ & $2,84 * *$ & $2,31 * *$ & $0,91 \mathrm{NS}$ \\
\hline $\mathrm{B} \times \mathrm{C}$ & $1,71 \mathrm{NS}$ & $2,15 \mathrm{NS}$ & $1,09 \mathrm{NS}$ & $0,71 \mathrm{NS}$ \\
\hline $\mathrm{A} \times \mathrm{B} \times \mathrm{C}$ & $0,72 \mathrm{NS}$ & $0,96 \mathrm{NS}$ & $1,37 \mathrm{NS}$ & $0,92 \mathrm{NS}$ \\
\hline$\% \mathrm{CV}$ parcela & 27,10 & 28,11 & 27,60 & 28,44 \\
\hline$\% \mathrm{CV}$ subparcela & 38,07 & 35,23 & 36,26 & 19,70 \\
\hline$\% \mathrm{CV}$ subsubparcela & 18,23 & 18,70 & 19,15 & 25,94 \\
\hline
\end{tabular}

Dados transformados em raiz $\mathrm{x}+1$. DAS - dias após semeadura. $* *$ significativo a $1 \%$ de probabilidade pelo teste $\mathrm{F}$. $*$ significativo a $5 \%$ de probabilidade pelo teste F. NS - não-significativo.

Observa-se pela Tabela 3 que, aos 45 DAS, a presença de $15 \mathrm{t} \mathrm{ha}^{-1}$ de palha reduziu em 46 e $62 \%$ o número de plantas de $I$. quamoclit e $M$. cissoides, respectivamente. No entanto, a presença de $20 \mathrm{t} \mathrm{ha}^{-1}$ reduziu em 82, 65, 62, 70,60 e $88 \%$ o número de plantas de I. quamoclit, I. purpurea, I. grandifolia, I. hederifolia, I. nil e $M$. cissoides, respectivamente, quando comparadas à ausência de palha.

Observou-se também, pela Tabela 3, que a densidade de $10 \mathrm{tha}^{-1}$ de palha reduziu o número de plantas de I. grandifolia e $M$. cissoides somente até os $15 \mathrm{DAS}$, pois ambas as espécies recuperaram-se nas demais avaliações, apresentando somente redução no número de plantas na presença de $20 \mathrm{t} \mathrm{ha}^{-1}$ de palha.

A tendência da camada de palha em reduzir o número de plantas daninhas também foi observada por Teasdale et al. (1991), que conseguiram reduzir em até $78 \%$ o número de plantas daninhas com cobertura de palha superior a $3 \mathrm{t} \mathrm{ha}^{-1}$. Martins et al. (1999) também 
constataram reduções significativas do número de plantas daninhas de Ipomoea grandifolia, $D$. horizontalis, B. plantaginea e B. decumbens ao utilizarem como densidade máxima $15 \mathrm{t} \mathrm{ha}^{-1}$ de palha de cana-de-açúcar.

Os baixos números de plantas daninhas encontrados em áreas cobertas por palha podem estar relacionados com a qualidade do comprimento de luz e a temperatura (Taylorson \& Borthwick, 1969; Fener, 1980) ou com o impedimento físico formado pela camada de palha sobre o desenvolvimento dos cotilédones, que não conseguem ultrapassar a barreira da palha e morrem antes de iniciar o processo fotossintético (Pitelli, 1995).

Quanto às oscilações térmicas no solo (Tabela 4), observou-se que a temperatura diminuiu com o aumento das densidades de palha, sendo a máxima redução observada de 13,2 e $6,2 \%$, comparando a ausência e presença de $20 \mathrm{t} \mathrm{ha}^{-1}$ de palha nas profundidades de 5 e $10 \mathrm{~cm}$, respectivamente. Nessa densidade, pode-se deduzir que os baixos números de plantas daninhas sejam também devido à temperatura, pois, segundo Egley \& Duke (1985) e Velini \& Negrissoli (2000), a amplitude térmica na superfície do solo pode interferir na germinação de muitas espécies.

Tabela 3 - Números de plantas de IPOQU, PHBPU, IAOGR, IPOHF, IPONI e MRRCI obtidos no desdobramento da interação entre as densidades de palha e as espécies. Jaboticabal, 2000

\begin{tabular}{|c|c|c|c|c|c|c|c|}
\hline \multirow{2}{*}{ DAS } & \multirow{2}{*}{$\begin{array}{c}\text { Densidade } \\
\left(\mathrm{t} \mathrm{ha}^{-1}\right)\end{array}$} & \multicolumn{6}{|c|}{ Espécie } \\
\hline & & IPOQU & PHBPU & IAOGR & IPOHF & IPONI & MRRCI \\
\hline \multirow{5}{*}{15} & 0 & $\begin{array}{c}5,92 \mathrm{Abc} \\
(35,5)\end{array}$ & $\begin{array}{c}4,37 \mathrm{Ad} \\
(18,4)\end{array}$ & $\begin{array}{c}6,67 \mathrm{Ab} \\
(46,0)\end{array}$ & $\begin{array}{c}8,34 \mathrm{Aa} \\
(69,9)\end{array}$ & $\begin{array}{c}5,56 \mathrm{Abc} \\
(30,2)\end{array}$ & $\begin{array}{c}5,52 \mathrm{Ac} \\
(31,3)\end{array}$ \\
\hline & 5 & $\begin{array}{c}5,90 \mathrm{Abc} \\
(33,9)\end{array}$ & $\begin{array}{c}4,32 \mathrm{Ad} \\
(17,7)\end{array}$ & $\begin{array}{c}6,53 \mathrm{Ab} \\
(44,6)\end{array}$ & $\begin{array}{c}7,77 \mathrm{ABa} \\
(59,8)\end{array}$ & $\begin{array}{c}5,54 \mathrm{Abc} \\
(29,9)\end{array}$ & $\begin{array}{c}4,84 \mathrm{ABcd} \\
(24,7)\end{array}$ \\
\hline & 10 & $\begin{array}{c}5,29 \mathrm{ABb} \\
(27,3)\end{array}$ & $\begin{array}{c}3,74 \mathrm{Ac} \\
(13,1)\end{array}$ & $\begin{array}{c}5,25 \mathrm{Bb} \\
(28,5)\end{array}$ & $\begin{array}{c}7,13 \mathrm{ABa} \\
(50,2)\end{array}$ & $\begin{array}{c}5,12 \mathrm{Ab} \\
(25,6)\end{array}$ & $\begin{array}{c}4,16 \mathrm{BCbc} \\
(17,3)\end{array}$ \\
\hline & 15 & $\begin{array}{c}4,52 \mathrm{Bb} \\
(20,2)\end{array}$ & $\begin{array}{c}3,40 \mathrm{ABbc} \\
(10,9)\end{array}$ & $\begin{array}{c}5,70 \mathrm{ABa} \\
(33,3)\end{array}$ & $\begin{array}{c}6,71 \mathrm{Ba} \\
(44,9)\end{array}$ & $\begin{array}{c}4,40 \mathrm{ABbc} \\
(18,9)\end{array}$ & $\begin{array}{c}3,31 \mathrm{Cc} \\
(11,1)\end{array}$ \\
\hline & 20 & $\begin{array}{c}1,92 \mathrm{Cc} \\
(3,6)\end{array}$ & $\begin{array}{c}2,44 \mathrm{Bbc} \\
(5,4)\end{array}$ & $\begin{array}{c}3,30 \mathrm{Cab} \\
(11,7)\end{array}$ & $\begin{array}{c}4,03 \mathrm{Ca} \\
(17,2)\end{array}$ & $\begin{array}{c}3,20 \mathrm{Bab} \\
(9,9)\end{array}$ & $\begin{array}{c}1,50 \mathrm{Dc} \\
(1,5)\end{array}$ \\
\hline \multirow{5}{*}{30} & 0 & $\begin{array}{c}6,24 \mathrm{Abc} \\
(39,2)\end{array}$ & $\begin{array}{c}4,36 \mathrm{Ad} \\
(18,3)\end{array}$ & $\begin{array}{c}6,92 \mathrm{Ab} \\
(49,5)\end{array}$ & $\begin{array}{c}8,80 \mathrm{Aa} \\
(78,3)\end{array}$ & $\begin{array}{c}5,55 \text { Acd } \\
(30,2)\end{array}$ & $\begin{array}{c}\text { 5,53Acd } \\
(31,0)\end{array}$ \\
\hline & 5 & $\begin{array}{c}6,08 \mathrm{Abc} \\
(36,1)\end{array}$ & $\begin{array}{c}4,34 \mathrm{Ad} \\
(18,0)\end{array}$ & $\begin{array}{c}6,51 \mathrm{ABb} \\
(44,3)\end{array}$ & $\begin{array}{c}8,04 \mathrm{ABa} \\
(64,2)\end{array}$ & $\begin{array}{c}5,45 \mathrm{Abcd} \\
(29,1)\end{array}$ & $\begin{array}{c}5,02 \mathrm{Acd} \\
(26,7)\end{array}$ \\
\hline & 10 & $\begin{array}{c}5,64 \mathrm{Ab} \\
(31,0)\end{array}$ & $\begin{array}{c}3,75 \mathrm{ABc} \\
(13,3)\end{array}$ & $\begin{array}{c}5,32 \mathrm{ABb} \\
(38,7)\end{array}$ & $\begin{array}{c}8,00 \mathrm{ABa} \\
(63,4)\end{array}$ & $\begin{array}{c}5,30 \mathrm{Ab} \\
(27,4)\end{array}$ & $\begin{array}{c}4,55 \mathrm{ABbc} \\
(21,0)\end{array}$ \\
\hline & 15 & $\begin{array}{l}4,94 \text { Acd } \\
(24,2)\end{array}$ & $\begin{array}{c}3,53 \mathrm{ABbcd} \\
(12,5)\end{array}$ & $\begin{array}{c}6,22 \mathrm{Bab} \\
(29,7)\end{array}$ & $\begin{array}{c}7,21 \mathrm{Ba} \\
(52,2)\end{array}$ & $\begin{array}{c}4,80 \mathrm{Aabc} \\
(22,7)\end{array}$ & $\begin{array}{c}3,52 \mathrm{Bd} \\
(12,9)\end{array}$ \\
\hline & 20 & $\begin{array}{c}2,61 \mathrm{Ba} \\
(6,6)\end{array}$ & $\begin{array}{c}2,99 \mathrm{Ba} \\
(9,4)\end{array}$ & $\begin{array}{c}3,96 \mathrm{Ca} \\
(16,7)\end{array}$ & $\begin{array}{c}4,37 \mathrm{Ca} \\
(20,8)\end{array}$ & $\begin{array}{c}3,42 \mathrm{Ba} \\
(11,7)\end{array}$ & $\begin{array}{c}1,86 \mathrm{Cb} \\
(3,2)\end{array}$ \\
\hline \multirow{5}{*}{45} & 0 & $\begin{array}{c}6,55 \mathrm{Ab} \\
(44,0)\end{array}$ & $\begin{array}{c}4,57 \mathrm{Ac} \\
(30,3)\end{array}$ & $\begin{array}{c}6,78 \mathrm{ABb} \\
(47,0)\end{array}$ & $\begin{array}{c}8,91 \mathrm{Aa} \\
(80,7)\end{array}$ & $\begin{array}{c}5,59 \mathrm{Abc} \\
(30,5)\end{array}$ & $\begin{array}{c}5,68 \mathrm{Abc} \\
(32,5)\end{array}$ \\
\hline & 5 & $\begin{array}{c}5,97 \mathrm{ABbc} \\
(35,5)\end{array}$ & $\begin{array}{c}4,39 \mathrm{Ad} \\
(18,4)\end{array}$ & $\begin{array}{c}6,88 \mathrm{Aab} \\
(49,3)\end{array}$ & $\begin{array}{c}8,14 \mathrm{Aa} \\
(66,0)\end{array}$ & $\begin{array}{c}5,43 \mathrm{Acd} \\
(28,8)\end{array}$ & $\begin{array}{c}\text { 4,99Acd } \\
(26,2)\end{array}$ \\
\hline & 10 & $\begin{array}{c}6,08 \mathrm{ABb} \\
(36,4)\end{array}$ & $\begin{array}{c}4,01 \mathrm{ABd} \\
(15,2)\end{array}$ & $\begin{array}{c}5,50 \mathrm{Bbc} \\
(31,7)\end{array}$ & $\begin{array}{c}8,10 \mathrm{Aa} \\
(65,0)\end{array}$ & $\begin{array}{c}5,37 \mathrm{Abc} \\
(28,2)\end{array}$ & $\begin{array}{c}4,62 \mathrm{ABcd} \\
(21,8)\end{array}$ \\
\hline & 15 & $\begin{array}{c}4,85 \mathrm{Bc} \\
(23,9)\end{array}$ & $\begin{array}{c}3,54 \mathrm{ABd} \\
(11,9)\end{array}$ & $\begin{array}{c}6,26 \mathrm{ABb} \\
(39,5)\end{array}$ & $\begin{array}{c}7,69 \mathrm{Aa} \\
(59,7)\end{array}$ & $\begin{array}{c}5,04 \mathrm{Abc} \\
(25,1)\end{array}$ & $\begin{array}{c}3,44 \mathrm{Bd} \\
(12,3)\end{array}$ \\
\hline & 20 & $\begin{array}{c}2,86 \mathrm{Cbc} \\
(8,1)\end{array}$ & $\begin{array}{c}2,81 \mathrm{Bbc} \\
(7,4)\end{array}$ & $\begin{array}{c}4,05 \mathrm{Cab} \\
(18,3)\end{array}$ & $\begin{array}{c}4,68 \mathrm{Ba} \\
(24,2)\end{array}$ & $\begin{array}{c}3,50 \mathrm{Bab} \\
(12,1)\end{array}$ & $\begin{array}{c}2,07 \mathrm{Cc} \\
(4,2)\end{array}$ \\
\hline
\end{tabular}

Dados transformados em raiz $\mathrm{x}+1$. Dados reais entre parênteses $(\%)$. DAS - dias após semeadura. Densidades $\mathrm{x}$ espécie - letras maiúsculas. Espécies x densidade - letras minúsculas. 
Tabela 4 - Valores médios e desvios-padrão da temperatura do solo aos cinco e dez centímetros de profundidade, entre oito e quinze dias após a semeadura das plantas daninhas

\begin{tabular}{|c|c|c|c|c|}
\hline \multirow{2}{*}{$\begin{array}{c}\text { Palha } \\
\left(\mathrm{t} \mathrm{ha}^{-1}\right)\end{array}$} & \multicolumn{4}{|c|}{ Temperatura do solo } \\
\cline { 2 - 5 } & Média $\left({ }^{\circ} \mathrm{C}\right)$ & Desvio-padrão & Média $\left({ }^{\circ} \mathrm{C}\right)$ & Desvio-padrão \\
\cline { 2 - 5 } & 24,42 & 6,29 & 22,36 & 3,62 \\
5 & 22,80 & 4,18 & 21,65 & 2,51 \\
10 & 21,72 & 2,69 & 21,10 & 1,60 \\
15 & 21,34 & 2,06 & 20,99 & 1,32 \\
20 & 21,20 & 1,66 & 20,97 & 1,09 \\
\hline
\end{tabular}

* As médias referem-se às temperaturas determinadas diariamente às 7h30, $13 \mathrm{~h} 30$ e 16h30.

Portanto, seja por motivo de temperatura ou simplesmente impedimento físico, a densidade de $20 \mathrm{t} \mathrm{ha}^{-1}$ de palha de cana-de-açúcar, em relação à ausência de palha, reduziu em mais de $60 \%$ o número de plantas de $M$. cissoides, Ipomoea quamoclit, I. purpurea, I. grandifolia, I. hederifolia e I. nil.

\section{AGRADECIMENTOS}

Ao Prof. Dr. José Carlos Barbosa, da Faculdade de Ciências Agrárias e Veterinárias de Jaboticabal, pela colaboração na análise estatística.

\section{LITERATURA CITADA}

ALMEIDA, F. S.; RODRIGUES, B. N. Guia de herbicidas - contribuição para o uso adequado em plantio direto e convencional. Londrina: Fundação Instituto Agronômico do Paraná, 1985. 482 p.

EGLEY, G. H.; DUKE, S. Physiology of weed seed dormancy and germination. In: DUKE, S. O. Weed physiology. I- reproduction and ecophysiology. Florida: CRC Press, 1985. p. 27-64.

FENER, M. Germination tests of thirty-two East African weed species. Weed Res., v. 20, p. 135-138, 1980.
MARTINS, D. et al. Emergência em campo de dicotiledôneas infestantes em solo coberto com palha de cana-de-açúcar. Planta Daninha, v. 17, n. 1, p. 151-161, 1999.

MEDINA MELENDEZ, J. A. Efeito da cobertura do solo no controle de plantas daninhas na cultura do pepino (Cucumis sativus L.). Piracicaba: Escola Superior de Agricultura "Luiz de Queiroz", 1990. 104 p. Dissertação (Mestrado em Agronomia) - Escola Superior de Agricultura "Luiz de Queiroz", 1990.

PITELLI, R. A. Dinâmica de plantas daninhas no sistema de plantio direto. In: CONGRESSO BRASILEIRO DA CIÊNCIA DAS PLANTAS DANINHAS, 1995,

Florianópolis. Palestras... Florianópolis: Sociedade Brasileira da Ciência das Plantas Daninhas, 1995. p. 5-12.

TAYLORSON, R. B.; BORTHWICK, H. A. Light filtration by foliar canopies: significance for light-controlled weed seed germination. Weed Sci., v. 17, n. 1, p. 48-51, 1969.

TEASDALE, J. R.; BESTE, C. E.; POTTS, W. E. Response of weeds to tillage and cover crop residue. Weed Sci., v. 39, p. 195-99, 1991.

VELINI, E. D.; NEGRISSOLI, E. Controle de plantas daninhas em cana crua. In: CONGRESSO BRASILEIRO DA CIÊNCIA DAS PLANTAS DANINHAS, 22., 2000, Foz do Iguaçu. Anais... Foz do Iguaçu: 2000. p. 148-164. 